\title{
A Class of Bessel Summations
}

\author{
By M. L. Glasser*
}

\begin{abstract}
A number of infinite series for which the general term contains the product of two Bessel functions are summed in closed form.
\end{abstract}

In this note we consider series of the form

$$
S(x)=\sum_{n=1}^{\infty} \frac{\chi(n)}{n^{\alpha}} J_{\mu}\left(n^{\lambda} x\right) J_{\nu}\left(n^{\lambda} x\right), \quad \mu, \nu>-\frac{1}{2},
$$

which were suggested by DeDoelder [1], where $\chi(n)$ is a character corresponding to the elementary Dirichlet series

$$
L(s ; \chi)=\sum_{1}^{\infty} \chi(n) n^{-s}
$$

These quantities have a host of useful properties which are concisely summarized in [3]. In particular, $L(s ; \chi)$ is an entire function except for the trivial character $\chi(n) \equiv 1$ for which $L(s ; 1)=\zeta(s)$ has a simple pole at $s=1$. We shall express $S(x)$ as a power series, indicate various sets of parameters for which it can be summed in closed form, and give some examples.

The Mellin transform of $S(x)$ is [2]

$$
\begin{aligned}
& \int_{0}^{\infty} x^{s-1} S(x) d x \\
& =\frac{1}{2} \frac{2^{s} L(\alpha+\lambda s ; \chi) \Gamma(1-s) \Gamma\left(\frac{1}{2} \mu+\frac{1}{2} \nu+\frac{1}{2} s\right)}{\Gamma\left(\frac{1}{2} \nu-\frac{1}{2} \mu-\frac{1}{2} s+1\right) \Gamma\left(\frac{1}{2} \mu-\frac{1}{2} \nu-\frac{1}{2} s+1\right) \Gamma\left(\frac{1}{2} \mu+\frac{1}{2} \nu-\frac{1}{2} s+1\right)},
\end{aligned}
$$

where the interchange in the order of summation and integration requires that

$$
\begin{aligned}
& C_{0}<\operatorname{Re} s<1, \\
& C_{0}=\max \left\{-(\mu+\nu), \begin{array}{c}
-\alpha / \lambda, \\
(1-\alpha) / \lambda
\end{array}\right\}, \quad \begin{array}{l}
\chi \neq 1, \\
\chi \equiv 1 .
\end{array}
\end{aligned}
$$

Now, by applying the Mellin inversion formula, we have the integral representation

$$
S(x)=\frac{1}{2} \int_{c-i \infty}^{c+i \infty} \frac{(x / 2)^{-s} L(\alpha+\lambda s ; \chi) \Gamma(1-s) \Gamma\left(\frac{1}{2} \mu+\frac{1}{2} \nu+\frac{1}{2} s\right)}{\Gamma\left(\frac{1}{2} \nu-\frac{1}{2} \mu-\frac{1}{2} s+1\right) \Gamma\left(\frac{1}{2} \mu-\frac{1}{2} \nu-\frac{1}{2} s+1\right) \Gamma\left(\frac{1}{2} \mu+\frac{1}{2} \nu-\frac{1}{2} s+1\right)} \frac{d s}{2 \pi i},
$$

where $C_{0}<c<1$. The integrand in (5) is meromorphic with poles to the left of the contour:

$$
s_{-1}=(1-\alpha) / \lambda \quad(\text { if } \chi \equiv 1), \quad s_{k}=-2 k-\mu-\nu, \quad k=0,1,2, \ldots
$$

Received September 3, 1980; revised January 30, 1981.

1980 Mathematics Subject Classification. Primary 33A40.

- This work was supported in part by the National Science Foundation grant \# MCS-78-0400. 
If $\operatorname{Re} \lambda \leqslant 1$, and $0 \leqslant x<2$, the integrand in (5) decays sufficiently rapidly into the left-hand plane that the contour may be closed and the integral be evaluated by the residue theorem. For simplicity we shall assume that for $\chi \equiv 1, s_{-1} \neq s_{k}$ for any $k$, or

$$
(\alpha / \lambda)-\mu-\nu \neq(1 / \lambda)+2 k, \quad k=0,1,2, \ldots,
$$

to ensure that there are only first order poles, and we thus have

$$
\begin{aligned}
S(x)= & \frac{\delta}{2 \lambda} \frac{\Gamma\left(1+\frac{\alpha-1}{\lambda}\right) \Gamma\left(\frac{1}{2} \mu+\frac{1}{2} \nu+\frac{1-\alpha}{2 \lambda}\right)(x / 2)^{(\alpha-1) / \lambda}}{\Gamma\left(\frac{1}{2} \nu-\frac{1}{2} \mu+\frac{\alpha-1}{2 \lambda}+1\right) \Gamma\left(\frac{1}{2} \mu-\frac{1}{2} \nu+\frac{\alpha-1}{2 \lambda}+1\right) \Gamma\left(\frac{1}{2} \mu+\frac{1}{2} \nu+\frac{\alpha-1}{2 \lambda}+1\right)} \\
& +\sum_{k=0}^{\infty} \frac{(-1)^{k}}{k !} \frac{L(\alpha-2 \lambda k-\lambda(\mu+\nu) ; \chi) \Gamma(1+2 k+\mu+\nu)(x / 2)^{2 k+\mu+\nu}}{\Gamma(\nu+k+1) \Gamma(\mu+k+1) \Gamma(\mu+\nu+k+1)}
\end{aligned}
$$

where $\delta$ is unity if $\chi \equiv 1$, and zero otherwise. Equation (8) is the desired power series expansion for $S(x)$; for $x \neq 1$ it can also be derived by inserting the power series for the Bessel function product into (1) and interchanging the order of summation. In the derivation we have assumed that $0<x<2$, but the actual range of validity may be much larger.

Whenever the series in (8) terminates we have $S(x)$ expressed in closed form and it is reasonable to conjecture that the converse is also the case. As is the case for the Riemann-Zeta functions, all the elementary Dirichlet series have "trivial" zeros when the argument is an (even or odd) negative integer. In view of condition (7) this cannot happen for the Dirichlet series in (8) unless $\lambda=1$. Hence, since the series does not terminate unless $\lambda=1$, we shall restrict further attention to this case and look at some examples.

Let $p$ be a positive integer and let $\chi_{p}$ be a primitive character [3] for which $\chi_{p}(n)= \pm 1,0$ and

$$
\begin{aligned}
& \chi_{p}(1)=1, \quad \chi_{p}(n)=0 \quad \text { if }(p, n) \neq 1, \\
& \chi_{p}(n)=\chi_{p}(n+p), \quad \chi_{p}(m n)=\chi_{p}(m) \chi_{p}(n) .
\end{aligned}
$$

Here $(p, n)$ denotes the greatest common divisor of $p$ and $n$. We write $L\left(s ; \chi_{p}\right)=$ $L_{ \pm p}(s)$ according as $\chi_{p}(p-1)= \pm 1$. It then follows from the functional equations satisfied by these Dirichlet series that for $k=1,2,3, \ldots$,

$$
L_{p}(-2 k)=L_{-p}(1-2 k)=0 \text {. }
$$

Consequently, $S(x)$ can be expressed in closed form in the two cases

$$
\begin{array}{ll}
\text { A: } \chi_{p}(p-1)=1, & \alpha-\mu-\nu \text { even, } \\
\text { B: } \chi_{p}(p-1)=-1, & \alpha-\mu-\nu \text { odd. }
\end{array}
$$

Two interesting examples are

$$
\begin{aligned}
& \sum_{1}^{\infty} \chi_{p}(n) J_{\mu}(n x) J_{m-\mu}(n x)=-\frac{\delta m, 0}{p} \sum_{l=1}^{p} l \chi_{p}(l), \quad \chi \neq 1 \\
&\left(m=0,1,2, \ldots ;-\frac{1}{2}<\mu<m+\frac{1}{2}\right), \\
& \sum_{1}^{\infty} \chi_{p}(n) J_{\mu}(n x) J_{m-\mu}(n x)=0,
\end{aligned}
$$


where $x_{p}(p-1)=+1$ or -1 , respectively. For the trivial character $\chi \equiv 1$, we easily find

$$
\begin{aligned}
& \sum_{n=1}^{\infty} \frac{J_{\mu}(n x) J_{\nu}(n x)}{n^{\alpha}} \\
&= \frac{1}{2} \frac{\Gamma(\alpha) \Gamma\left\{\frac{1}{2}(\mu+\nu-\alpha+1)\right\}(x / 2)^{\alpha-1}}{\Gamma\left\{\frac{1}{2}(\nu-\mu+\alpha+1)\right\} \Gamma\left\{\frac{1}{2}(\mu-\nu+\alpha+1)\right\} \Gamma\left\{\frac{1}{2}(\mu+\nu+\alpha+1)\right\}} \\
&+\sum_{k=0}^{(\alpha-\mu-\nu) / 2} \frac{(-1)^{k}}{k !} \frac{\zeta(\alpha-\mu-\nu-2 k) \Gamma(\mu+\nu+1+2 k)(x / 2)^{2 k+\mu+\nu}}{\Gamma(\nu+k+1) \Gamma(\mu+k+1) \Gamma(\mu+\nu+k+1)}, \\
&(\alpha-\mu-\nu \text { even }),
\end{aligned}
$$

where the sum is empty if $\alpha-\mu-\nu \leqslant-2$. For the alternating character, we see that

$$
\sum_{n=1}^{\infty}(-1)^{n-1} \frac{J_{\mu}(n x) J_{\nu}(n x)}{n^{\mu+\nu}}=\frac{(x / 2)^{\mu+\nu}}{2 \Gamma(\mu+1) \Gamma(\nu+1)},
$$

and for the character $\chi_{4}$ we have

$$
\sum_{n=0}^{\infty} \frac{(-1)^{n} J_{2 p+1}^{2}((2 n+1) x)}{(2 n+1)}=0, \quad p=0,1,2, \ldots
$$

We conclude by noting that when $\mu$ and $\nu$ are half integers the Bessel functions are trigonometric, and we obtain a host of new Fourier series. For example,
a) $\chi_{p}(p-1)=1$,$$
\sum_{n=1}^{\infty} \chi_{p}(n) \frac{\operatorname{Cos}(n x)}{n^{2}}=L_{p}(2)-\frac{1}{4} L_{p}(0) x^{2}
$$
b) $\chi_{p}(p-1)=-1$,$$
\sum_{n=1}^{\infty} x_{p}(n) \frac{\operatorname{Cos} n x}{n}=L_{-p}(1) \text {, }
$$
c)

$$
\sum_{n=1}^{\infty} \chi_{p}(n) \frac{\operatorname{Cos} n x}{n^{3}}=L_{-p}(3)-\frac{1}{4} L_{-p}(0) x^{2} \text {. }
$$

Thus, for $p=3$, (16b) gives

$$
T(x)=\operatorname{Cos} x-\frac{\operatorname{Cos} 2 x}{2}+\frac{\operatorname{Cos} 4 x}{4}-\frac{\operatorname{Cos} 5 x}{5}+\ldots=\pi / 3 \sqrt{3} .
$$

These summations are only valid in this case for $0<x<2$. The values of the series outside this range must be found by other means. In fact, it is easily verified that the sum of the series in (17) is the periodic extension of

$$
T(x)= \begin{cases}\pi / 3 \sqrt{3}, & 0<|x|<2 \pi / 3 \\ -2 \pi / 3 \sqrt{3}, & 2 \pi / 3<|x|<\pi \\ -\pi / 6 \sqrt{3}, & |x|=2 \pi / 3\end{cases}
$$

Department of Mathematics and Computer Science

Clarkson College

Potsdam, New York 13676

1. P. J. DeDoelder, SIAM Rev., v. 21, 1979, Prob. 79-12, p. 395.

2. A. Erdély et AL., Tables of Integral Transforms, Vol. 1, McGraw-Hill, New York, 1954.

3. I. J. ZuCKer \& M. M. Robertson, J. Phys. A, v. 9, 1976, p. 1207. 